\title{
Role of microRNAs in cardiac development and disease (Review)
}

\author{
JING TIAN, XINJIANG AN and LING NIU \\ Department of Cardiology, Xuzhou Children's Hospital, Xuzhou, Jiangsu 221002, P.R. China
}

Received June 13, 2016; Accepted November 25, 2016

DOI: $10.3892 /$ etm.2016.3932

\begin{abstract}
Heart disease-related deaths are the highest in most societies and congenital heart diseases account for approximately $40 \%$ of prenatal deaths and over $20 \%$ of mortality in the first few months after birth. Congenital heart disease affects approximately $1 \%$ of all newborns and is the causative factor for more deaths within the first year of life as compared to all other genetic defects. Advances in treatment approaches increased life expectancy and led to an expansion of adult population with clinical manifestation of congenital heart defects in up to $90 \%$ of the children born with congenital heart diseases. Regulation of cardiac gene expression involves multiple independent enhancers that play a critical role in maintaining a restricted and specific pattern of gene expression in the heart. Cardiac transcriptional pathways are intimately regulated by microRNAs (miRNAs), which are small, regulatory RNAs, approximately 22 nucleotides in length, also coded by specific genes. These miRNAs act as suppressors of gene expression by inhibiting translation and/or promoting degradation of target protein-coding mRNAs. There are several miRNAs involved in the development of heart and dysregulation of specific miRNAs is associated with congenital and other cardiac defects. Stress responsive cardiac hypertrophy is orchestrated among other factors, by specific miRNAs. miRNAs such as miR-499 are considered useful as biomarkers of a given heart disease. Therapeutic application of miRNAs is also envisaged considering the small size and specific effects of these molecules. In this review, we addressed different roles of miRNAs in the development and diseases of the heart.
\end{abstract}

\section{Contents}

1. Introduction

2. Role of miRNAs in normal heart development and function

Correspondence to: Dr Xinjiang An, Department of Cardiology, Xuzhou Children's Hospital, 18 Sudibei Road, Xuzhou, Jiangsu 221002, P.R. China

E-mail: anxinjian001@163.com

Key words: microRNA, cardiac disease, cardiomyopathy, myocardial infarction, transcriptional pathways, gene expression
3. Congenital cardiac defects and altered miRNAs

4. Importance of miRNAs in cardiac remodeling and cardiac hypertrophy

5. miRNAs as therapeutic targets and biomarkers

6. Conclusions

\section{Introduction}

Heart disease-related deaths are the highest in most societies and congenital heart diseases have childhood origins, necessitating their early diagnosis and treatment. In fact, congenital heart diseases account for approximately $40 \%$ of prenatal deaths and over $20 \%$ of mortality in the first few months after birth (1). Congenital heart disease affects approximately $1 \%$ of all newborns and is the causative factor for more deaths within the first year of life as compared to all other genetic defects (2). A complete cure of congenital heart defect during early childhood is rare, but advances in treatment approaches have increased life expectancy and led to an expansion of adult population with clinical manifestation of congenital heart defects in up to $90 \%$ of the children born with congenital heart diseases (3). During development, at the embryonic stage itself, formation of the functional heart is of utmost importance and the process of cardiac tissue formation is a highly complex multi-cell lineage differentiation process. The uninterrupted contractile function of heart from embryonic stage till the end of life is critical for survival of the organism. The most common congenital defects are seen in heart development that lead to a wide variety of abnormalities such as, arrhythmias, cardiomyopathies, heart failure, and malformed valves all leading to sudden death (4). Development of heart and cardiac function is regulated by multiple transcription factors, signaling proteins and complexes that orchestrate cardiac morphogenesis and myogenesis and control cardiac contractility (5). Several complex genetic pathways are linked to heart development and pathology and a clear understanding of these players at molecular level is essential in improving the prognosis of patients with cardiac abnormalities (6). Regulation of cardiac gene expression involves multiple independent enhancers that play a critical role in maintaining a restricted and specific pattern of gene expression in the heart. It is now recognized that cardiac transcriptional pathways are intimately regulated by microRNAs (miRNAs) (7). miRNAs are a class of small, regulatory RNAs, approximately 22 nucleotides in length at mature stage and are evolutionarily conserved and also coded by specific genes. These miRNAs normally function to 
suppress expression of genes they target by inhibiting translation and/or promoting degradation of target protein-coding mRNA by base pairing $(8,9)$. Considering that the miRNAs play their role post-transcriptionally by adding another layer of regulation of cardiac gene expression, they are proposed to act as 'rheostats' and 'switches' for controlling various aspects of cardiac development, function, and their dysregulation can lead to cardiac abnormalities including hypertrophy, arrhythmia and ischemia $(10,11)$. Since their discovery 25 years ago, $>1,400$ miRNAs have been identified in mammals (12) and are proposed to regulate the expression of $>50 \%$ of all protein coding genes (13). It has been reported that the expression of several miRNAs change in diseased hearts, emphasizing their role in cardiac disease (14). These findings are corroborated by experimental studies on animals addressing specific roles of different miRNAs (15-17).

\section{Role of miRNAs in normal heart development and function}

Genomic sequences coding for miRNAs are located in the intergenic, intronic, and exonic regions in chromosomes. Intergenic miRNAs are produced from their own transcriptional units whereas intronic and exonic miRNAs are transcribed and expressed along their host genes. A subset of intronic miRNAs are transcribed in the opposite orientation of the host genes and these miRNAs have their own cis-regulatory elements for expression control (18). Biogenesis of miRNAs starts with transcription of the corresponding gene in the nucleus to generate primary miRNA, which is further processed to produce $\sim 70$ nucleotide long precursor miRNA, by a complex of Drosha and DiGeorge syndrome critical region 8 (DGCR8). Precursor miRNA exits the nucleus facilitated by exportin 5 in the nuclear membrane (19), and is cut by Dicer, a type of RNAse III endonuclease, to give rise to miRNA duplex, in the cytosol. A single arm of the resulting 22 -nucleotide duplex is taken selectively into the RNA-induced silencing complex (RISC), whereas the other stem arm is presumably degraded. The miRNA-loaded RISC targets the mRNAs, by complementary base pairing the target sequence(s) within the $3^{\prime}$ untranslated region. A perfect or near-perfect complementary base pairing between RISC-bound miRNA and targeted mRNA leads to rapid degradation of the targeted mRNA. However, in most cases, animal miRNAs are only imperfectly complementary to their targeted mRNAs, resulting in suppressed translation from that mRNA or sequestration of the targeted mRNA to cytoplasmic P-bodies (20). The significance of miRNAs in development in general and specifically for cardiac development was realized in gene deletion experiments in mice and zebrafish, where Dicer gene is deleted. These animals suffered arrested development from gastrulation stage in association with almost total lack of miRNAs (21). Similarly animals with mutated Dicer showed abnormal somitogenesis and heart development (22). Tissue specific deletion of Dicer in mouse heart, employing Cre-Lox system, under the control of the postnatally expressed $\alpha$-myosin heavy chain promoter, led to deranged expression of cardiac contractile proteins and significant sarcomere disarray, in association with greatly decreased cardiac function. These hearts rapidly developed dilated cardiomyopathy and heart failure after birth (23). The abnormal heart function seen in Dicer mutant mice closely resembles the human dilated cardiomyopathy and heart failure and in fact, failing human hearts are shown to have low levels of Dicer protein, suggesting an important role for miRNAs in dilated cardiomyopathies and heart failure in patients (23). On the other hand, deletion of Dicer during mouse heart development, using Cre-recombinase under the control of Nkx2.5 promoter, led to embryonic lethality with defective heart morphogenesis (15) and this indicated the differential role of miRNAs during and after the development of the heart.

Studies on individual miRNAs expressed in heart revealed important and critical contribution of many of these miRNAs during heart development (Fig. 1). Thus miR-1 and miR-133 are experimentally verified to play a role in the cardiac development. These miRNAs are produced from the same polycistronic transcripts, encoded by two separate genes. In mice miR-1-1 and miR-133a-2 are clustered on chromosome 2, whereas miR-1-2 and miR-133a-1 are clustered on chromosome 18 (24). Expression of these miRNAs is regulated by muscle transcriptional networks, consisting of serum response factor and myocardin for cardiac muscle expression and MyoD and myocyte enhancer factor 2 for skeletal muscle expression (25). Even though miR-1-1 and miR-1-2 are encoded by separate genes, they have identical nucleotide sequence and thus appear to target the same mRNAs. However, miR-1-2 null mice in utero display pericardial edema, which is consistent with embryonic myocardial dysfunction and it appears that miR-1-2 has non-redundant roles with miR-1-1 in the heart, even though these two miRNAs have overlapping expression patterns and sequences (24). There appears to be a fine balance of the effects of these miRNAs as their excess activity or loss of function can be detrimental to the development and function of heart. Thus, miR-1 overexpression in the embryonic heart blocks expansion of ventricular myocardium by inhibiting cardiomyocyte proliferation (25) and also injection of Xenopus embryos with miR-1 arrests the cardiac development (24). On the other hand, targeted deletion of miR-1-2 was found to cause $\sim 50 \%$ embryonic lethality in mice because of ventricular septal defects, with the remaining surviving mice with the deletion facing mortality at later stage due to conduction system defects (15).

Understanding how exactly the miRNAs influence heart development is critical. Although miR-1 can potentially target several genes in the heart, one important validated target is Hand 2 cardiac transcription factor. Thus, deletion of Hand2 leads to similar ventricular myocyte developmental problems (26) as miR-1 over-production, which also reduces expression of Hand2 (25). Expression of miR-133a-1/miR-1-2 and miR-133a-2/miR-1-1 is found throughout the ventricular myocardium and also in interventricular septum from embryonic stage E8.5 until adulthood (27). Even though deletion of either miR-133a-1 or miR-133a-2 has no obvious deleterious effects on the heart, loss of both the miRs leads to ventricular septal defects and chamber dilatation resulting in late embryonic and neonatal lethality (28). Among the other miRNAs, miR-196a, which is found in fetal human heart is known to regulate HOXB8-Shh signaling, that is essential 


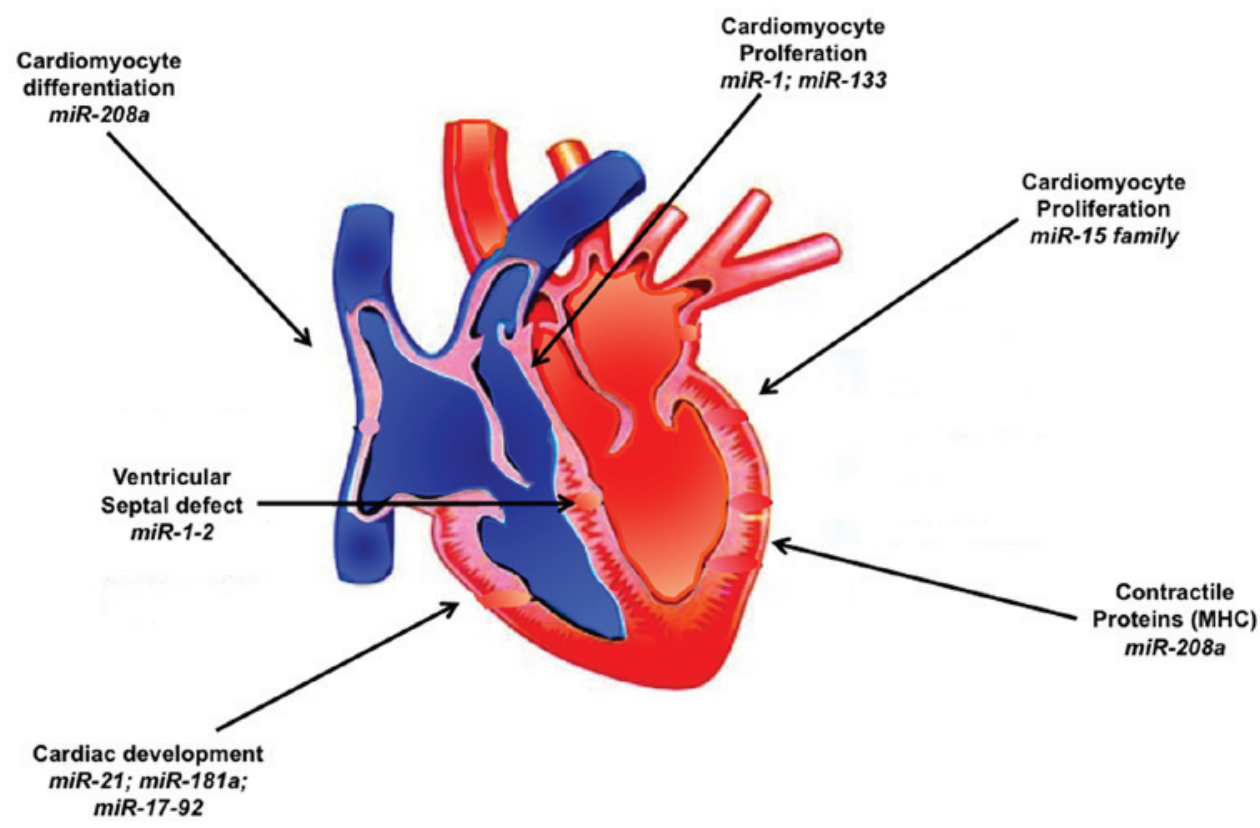

Figure 1. Involvement of microRNAs (miRNAs) in cardiac development and function. Several types of miRNAs are known to participate in the regulation of heart development from embryonic stage to the adult form and also in the normal function and stress response of the heart.

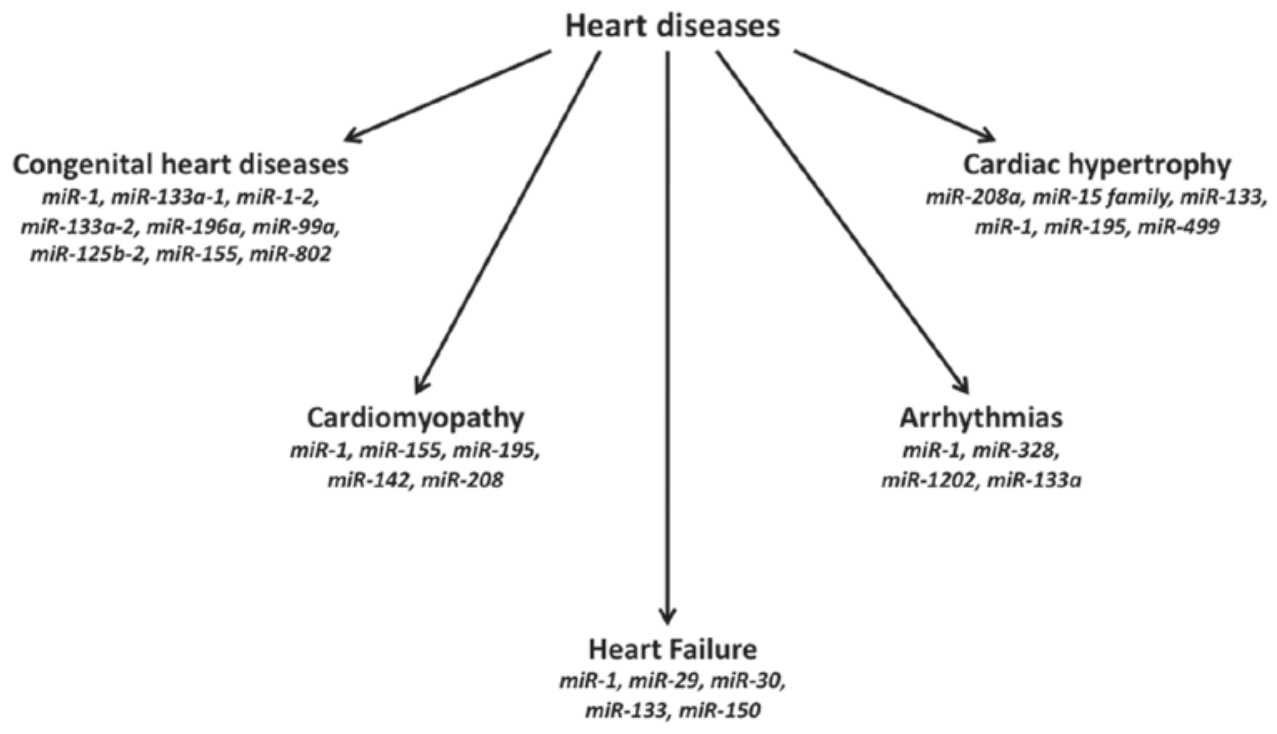

Figure 2. Involvement of microRNAs (miRNAs) in cardiovascular diseases. Besides participating in the development of heart, miRNAs are also implicated in multiple cardiac defects. Either overexpression or loss of expression of certain miRNAs is associated with congenital cardiac defects. Stress induced cardiac hypertrophy response is controlled by a large set of miRNAs.

for cardiac septation, outflow tract morphogenesis as well as valve formation (29).

\section{Congenital cardiac defects and altered miRNAs}

Several congenital cardiac defects have been found to be associated with altered expression of different miRNAs (Fig. 2). The hearts of patients with the most common genetic defect leading to cardiac abnormalities, the trisomy 21 (Down syndrome), contain an extra Hsa21 chromosome, and were found to overexpress 5 miRs: miR-99a, let-7c, miR-125b-2, miR-155, and miR-802, present on human chromosome 21 (30). It has been observed that $\sim 61$ miRNAs, which target the networks of cardiac development, show altered expression in the right ventricular myocardium of children with non-syndromic tetralogy of Fallot (31). In another condition of human fetal single ventricle malformation, 38 miRNAs were found to be downregulated and 10 miRNAs showed upregulation in cardiac tissues, as compared to normal control cardiac tissue (32). Patients suffering from DG syndrome, which is caused by a deletion of the DGCR8 on chromosome 22 (22q11.2), develop congenital heart disease. This deletion causes loss of a component of the RISC, thereby leading to impaired miRNA expression, which can potentially contribute to congenital heart defects (33). As this syndrome results from haploinsufficiency of the 22q11.2 locus, the 
possibility that disturbances in miRNA expression potentially contribute to the gene dosage sensitivity of this disease. It is interesting to note that while many miRNAs can be perturbed with minimal effects on phenotype under normal conditions, the same miRNA disturbances can have profound impact on phenotype under stress conditions (10). The apparent minimal effects of miRNAs under non-stress conditions as compared to their specific involvement during remodeling responses of diseased tissues make miRNAs attractive therapeutic targets for inactivating disease-inducing miRNAs with little or no off-target effects on normal non-stress tissues. Specific expression patterns of miRNAs have been observed in several cardiac disorders, including hypertrophy, heart failure, ischemic cardiomyopathy, post-myocardial infarction (MI) remodelling (14,34-36). Cardiac injury following acute MI is known to increase the circulating levels of certain myocardial-derived miRNAs, such as miR-1, miR-133, miR-499 and miR-208, and it has been proposed that the specifically increased miRNAs can be useful as both diagnostic as well as prognostic biomarkers $(37,38)$.

\section{Importance of miRNAs in cardiac remodeling and cardiac hypertrophy}

Heart being a highly sensitive tissue to various stresses and external pathological stimuli, responds by undergoing extensive cardiac remodeling, which is essentially cardiac hypertrophy (39). In cardiac hypertrophy there is an increase in the size of cardiomyocyte and/or myofibrillar volume without any change in cardiomyocyte number. This elevated cell size and volume is needed to maintain normal cardiac output under stress conditions. There is a reactivation of fetal cardiac genes during cardiac hypertrophy, indicating that the cardiac genes involved in heart development before birth are redeployed during cardiac hypertrophic growth. It has been recognized that expression of several miRNAs is both up- and down-regulated (Fig. 2) in experimental models of cardiac hypertrophy and also in samples from failing human hearts $(35,40)$. It has been demonstrated that miR-195 expression is elevated during cardiac hypertrophy in human hearts and in mouse hypertrophic hearts and that miR-195 alone is sufficient to induce hypertrophic growth in cultured rat cardiomyocytes (40).

During heart development and in diseased hearts, miR-208a and miR-208b are differentially expressed coinciding with the expression of their host genes, Myh6 and Myh7, respectively. These miRNAs regulate the host gene switch during development and under stress conditions in a feedback manner $(16,41)$. Gain-of-function studies showed that cardiac specific overexpression of miR-208a alone is able to induce cardiac hypertrophy and cardiac conduction defects (41). Expression of the Myh7/miR-208b is regulated by miR-208a, which also acts upstream of Myh7b/miR-499 in adult hearts. Another important target of miR-208a is thyroid hormone receptor-associated protein 1, a key component of the thyroid hormone signal pathway and an important player in cardiac hypertrophy $(16,41)$. While miR-195, miR-214 or miR-21 are upregulated during cardiac hypertrophy, the expression of miR-1, miR-133a, miR-93 and miR-181 is downregulated $(17,42)$. Repression of miR-133a is found to be sufficient to lead to cardiac hypertrophy both in vivo and in vitro, probably through the upregulation of RhoA, Cdc42, and NELFA/Whsc2 (17). On the other hand, knockout of both miR-133a-1 and miR-133a-2, leads to partial embryonic lethality with the remaining living mutant mice showing cardiomyopathy but not cardiac hypertrophy (28), indicating different experimental approaches may lead to different and some times discrepant results. It has been shown that deregulation of miR-499, which controls the expression of sarcomeric genes, contributes to the pathogenesis of cardiac hypertrophy (43).

\section{5. miRNAs as therapeutic targets and biomarkers}

Many of the properties of miRNAs make them clinically relevant because miRNA expression is altered in diseased hearts, making miRNAs as potential biomarkers for the diagnosis of cardiovascular disease. Another important feature of miRNAs is their smaller size, which makes their in vivo delivery feasible. Besides, considering that single miRNAs target multiple mRNAs coding for different proteins of a common pathway, it becomes easier to efficiently control a given pathway or biological function with a single miRNA. However, exploitation of this property also calls for caution to avoid potential off-target activities of the chosen miRNAs. It has been proposed that miR-1 is a potential biomarker for early diagnosis of acute MI, as circulating miR-1 levels are markedly increased in these patients and the circulating miR-1 levels can also be used to differentiate between acute MI and other cardiac events such as angina pectoris $(44,45)$, non-acute MI (46), and other cardiovascular diseases (47). Appearance of miR-499 in the blood is considered to be an indicator of heart damage following acute MI. Plasma miR-499 levels are increased several-fold in acute MI patients but are below detection in other conditions such as congestive heart failure and acute coronary syndrome and also in normal controls (48). It is interesting to note that cardiospecific regenerative capacity of miR-499 has the ability to promote preferential differentiation of cardiac stem cells (CSCs) to mature functional cardiomyocytes. In human CSCs, miR-499 overexpression led to improved regenerative potential of miR-499-overexpressing cell grafts, increased myocardial tissue repair, and better restoration of heart mass (49). Without such miR-499 overexpression, transplants of normal CSC to post-MI myocardium lead to the generation of cardiomyocyte-like immature cells that failed to differentiate to mature cardiomyocytes.

\section{Conclusions}

Heart disease related deaths are the highest in most societies and congenital heart diseases account for a significant part, particularly in infants and children. Increased survival of the children with congenital heart defects with advances in medical technologies, led to elevated number of adults with heart diseases. Various types of miRNAs are implicated in the development of heart and dysregulation of specific miRNAs is associated with congenital and other cardiac defects. Normal cardiac function as well as stress responsive cardiac hypertrophy is controlled among other factors, by 
specific miRNAs. Recent studies strongly implicate certain miRNAs such as miR-499 as useful biomarkers of a given heart disease. Therapeutic application of miRNAs is also being envisaged. More work is needed for the effective use of miRNAs either as diagnostic and prognostic markers and as therapeutic agents.

\section{References}

1. Trojnarska O, Grajek S, Katarzyński S and Kramer L: Predictors of mortality in adult patients with congenital heart disease. Cardiol J 16: 341-347, 2009.

2. Rosamond W, Flegal K, Furie K, Go A, Greenlund K, Haase N, Hailpern SM, Ho M, Howard V, Kissela B, et al; American Heart Association Statistics Committee and Stroke Statistics Subcommittee: Heart disease and stroke statistics - 2008 update: A report from the American Heart Association Statistics Committee and Stroke Statistics Subcommittee. Circulation 117: e25-e146, 2008.

3. Khairy P, Ionescu-Ittu R, Mackie AS, Abrahamowicz M, Pilote L and Marelli AJ: Changing mortality in congenital heart disease. J Am Coll Cardiol 56: 1149-1157, 2010.

4. Bruneau BG: The developmental genetics of congenital heart disease. Nature 451: 943-948, 2008.

5. Olson EN: Gene regulatory networks in the evolution and development of the heart. Science 313: 1922-1927, 2006.

6. Olson EN: A decade of discoveries in cardiac biology. Nat Med 10: 467-474, 2004.

7. Wang DZ: MicroRNAs in cardiac development and remodeling. Pediatr Cardiol 31: 357-362, 2010.

8. Kiriakidou M, Tan GS, Lamprinaki S, De Planell-Saguer M, Nelson PT and Mourelatos Z: An mRNA m7G cap binding-like motif within human Ago2 represses translation. Cell 129: 1141-1151, 2007.

9. Humphreys DT, Westman BJ, Martin DI and Preiss T: MicroRNAs control translation initiation by inhibiting eukaryotic initiation factor 4E/cap and poly(A) tail function. Proc Natl Acad Sci USA 102: 16961-16966, 2005.

10. Liu N and Olson EN: MicroRNA regulatory networks in cardiovascular development. Dev Cell 18: 510-525, 2010.

11. Cai B, Pan Z and $\mathrm{Lu} \mathrm{Y}$ : The roles of microRNAs in heart diseases: A novel important regulator. Curr Med Chem 17: 407-411, 2010.

12. Rota R, Ciarapica R, Giordano A, Miele L and Locatelli F: MicroRNAs in rhabdomyosarcoma: Pathogenetic implications and translational potentiality. Mol Cancer 10: 120, 2011.

13. Bartel DP: MicroRNAs: Target recognition and regulatory functions. Cell 136: 215-233, 2009.

14. Thum T, Galuppo P, Wolf C, Fiedler J, Kneitz S, van Laake LW, Doevendans PA, Mummery CL, Borlak J, Haverich A, et al: MicroRNAs in the human heart: A clue to fetal gene reprogramming in heart failure. Circulation 116: 258-267, 2007.

15. Zhao Y, Ransom JF, Li A, Vedantham V, von Drehle M, Muth AN, Tsuchihashi T, McManus MT, Schwartz RJ and Srivastava D: Dysregulation of cardiogenesis, cardiac conduction, and cell cycle in mice lacking miRNA-1-2. Cell 129: 303-317, 2007.

16. van Rooij E, Sutherland LB, Qi X, Richardson JA, Hill J and Olson EN: Control of stress-dependent cardiac growth and gene expression by a microRNA. Science 316: 575-579, 2007.

17. Carè A, Catalucci D, Felicetti F, Bonci D, Addario A, Gallo P, Bang ML, Segnalini P, Gu Y, Dalton ND, et al: MicroRNA-133 controls cardiac hypertrophy. Nat Med 13: 613-618, 2007.

18. Bartel DP: MicroRNAs: Genomics, biogenesis, mechanism, and function. Cell 116: 281-297, 2004.

19. Yi R, Qin Y, Macara IG and Cullen BR: Exportin-5 mediates the nuclear export of pre-microRNAs and short hairpin RNAs. Genes Dev 17: 3011-3016, 2003.

20. Filipowicz W, Bhattacharyya SN and Sonenberg N: Mechanisms of post-transcriptional regulation by microRNAs: Are the answers in sight? Nat Rev Genet 9: 102-114, 2008.

21. Bernstein E, Kim SY, Carmell MA, Murchison EP, Alcorn H, Li MZ, Mills AA, Elledge SJ, Anderson KV and Hannon GJ: Dicer is essential for mouse development. Nat Genet 35: 215-217, 2003.

22. Wienholds E, Koudijs MJ, van Eeden FJ, Cuppen E and Plasterk RH: The microRNA-producing enzyme Dicer1 is essential for zebrafish development. Nat Genet 35: 217-218, 2003.
23. Chen JF, Murchison EP, Tang R, Callis TE, Tatsuguchi M, Deng Z, Rojas M, Hammond SM, Schneider MD, Selzman CH, et al: Targeted deletion of Dicer in the heart leads to dilated cardiomyopathy and heart failure. Proc Natl Acad Sci USA 105: 2111-2116, 2008.

24. Chen JF, Mandel EM, Thomson JM, Wu Q, Callis TE, Hammond SM, Conlon FL and Wang DZ: The role of microRNA-1 and microRNA-133 in skeletal muscle proliferation and differentiation. Nat Genet 38: 228-233, 2006.

25. Zhao Y, Samal E and Srivastava D: Serum response factor regulates a muscle-specific microRNA that targets Hand2 during cardiogenesis. Nature 436: 214-220, 2005.

26. Srivastava D, Thomas T, Lin Q, Kirby ML, Brown D and Olson EN: Regulation of cardiac mesodermal and neural crest development by the bHLH transcription factor, dHAND. Nat Genet 16: 154-160, 1997.

27. Liu N, Williams AH, Kim Y, McAnally J, Bezprozvannaya S, Sutherland LB, Richardson JA, Bassel-Duby R and Olson EN: An intragenic MEF2-dependent enhancer directs muscle-specific expression of microRNAs 1 and 133. Proc Natl Acad Sci USA 104: 20844-20849, 2007.

28. Liu N, Bezprozvannaya S, Williams AH, Qi X, Richardson JA, Bassel-Duby R and Olson EN: microRNA-133a regulates cardiomyocyte proliferation and suppresses smooth muscle gene expression in the heart. Genes Dev 22: 3242-3254, 2008.

29. Goddeeris MM, Rho S, Petiet A, Davenport CL, Johnson GA, Meyers EN and Klingensmith J: Intracardiac septation requires hedgehog-dependent cellular contributions from outside the heart. Development 135: 1887-1895, 2008

30. Latronico MV, Catalucci D and Condorelli G: MicroRNA and cardiac pathologies. Physiol Genomics 34: 239-242, 2008.

31. O'Brien JE Jr, Kibiryeva N, Zhou XG, Marshall JA, Lofland GK, Artman M, Chen J and Bittel DC: Noncoding RNA expression in myocardium from infants with tetralogy of Fallot. Circ Cardiovasc Genet 5: 279-286, 2012.

32. Yu ZB, Han SP, Bai YF, Zhu C, Pan Y and Guo XR: microRNA expression profiling in fetal single ventricle malformation identified by deep sequencing. Int J Mol Med 29: 53-60, 2012.

33. Omran A, Elimam D, Webster KA, Shehadeh LA and Yin F: MicroRNAs: A new piece in the paediatric cardiovascular disease puzzle. Cardiol Young 23: 642-655, 2013.

34. Ikeda S, Kong SW, Lu J, Bisping E, Zhang H, Allen PD, Golub TR, Pieske B and Pu WT: Altered microRNA expression in human heart disease. Physiol Genomics 31: 367-373, 2007.

35. Tatsuguchi M, Seok HY, Callis TE, Thomson JM, Chen JF, Newman M, Rojas M, Hammond SM and Wang DZ: Expression of microRNAs is dynamically regulated during cardiomyocyte hypertrophy. J Mol Cell Cardiol 42: 1137-1141, 2007.

36. van Rooij E, Sutherland LB, Thatcher JE, DiMaio JM, Naseem RH, Marshall WS, Hill JA and Olson EN: Dysregulation of microRNAs after myocardial infarction reveals a role of miR-29 in cardiac fibrosis. Proc Natl Acad Sci USA 105: 13027-13032, 2008.

37. Fichtlscherer S,Zeiher AM, Dimmeler S and Sessa WC: Circulating microRNAs: Biomarkers or mediators of cardiovascular diseases? Arterioscler Thromb Vasc Biol 31: 2383-2390, 2011.

38. Tijsen AJ, Pinto YM and Creemers EE: Circulating microRNAs as diagnostic biomarkers for cardiovascular diseases. Am J Physiol Heart Circ Physiol 303: H1085-H1095, 2012.

39. Hunter JJ and Chien KR: Signaling pathways for cardiac hypertrophy and failure. N Engl J Med 341: 1276-1283, 1999.

40. van Rooij E, Sutherland LB, Liu N, Williams AH, McAnally J, Gerard RD, Richardson JA and Olson EN: A signature pattern of stress-responsive microRNAs that can evoke cardiac hypertrophy and heart failure. Proc Natl Acad Sci USA 103: 18255-18260, 2006

41. Callis TE, Pandya K, Seok HY, Tang RH, Tatsuguchi M, Huang ZP, Chen JF, Deng Z, Gunn B, Shumate J, et al: MicroRNA-208a is a regulator of cardiac hypertrophy and conduction in mice. J Clin Invest 119: 2772-2786, 2009.

42. Sayed D, Hong C, Chen IY, Lypowy J and Abdellatif M: MicroRNAs play an essential role in the development of cardiac hypertrophy. Circ Res 100: 416-424, 2007.

43. Matkovich SJ, Hu Y, Eschenbacher WH, Dorn LE and Dorn GW II: Direct and indirect involvement of microRNA-499 in clinical and experimental cardiomyopathy. Circ Res 111: 521-531, 2012.

44. Li C, Fang Z, Jiang T, Zhang Q, Liu C, Zhang C and Xiang Y: Serum microRNAs profile from genome-wide serves as a fingerprint for diagnosis of acute myocardial infarction and angina pectoris. BMC Med Genomics 6: 16, 2013. 
45. Kuwabara Y, Ono K, Horie T, Nishi H, Nagao K, Kinoshita M Watanabe S, Baba O, Kojima Y, Shizuta S, et al: Increased microRNA-1 and microRNA-133a levels in serum of patients with cardiovascular disease indicate myocardial damage. Circ Cardiovasc Genet 4: 446-454, 2011.

46. Ai X, Curran JW, Shannon TR, Bers DM and Pogwizd SM: $\mathrm{Ca}^{2+} /$ calmodulin-dependent protein kinase modulates cardiac ryanodine receptor phosphorylation and sarcoplasmic reticulum $\mathrm{Ca}^{2+}$ leak in heart failure. Circ Res 97: 1314-1322, 2005.

47. Wang GK, Zhu JQ, Zhang JT, Li Q, Li Y, He J, Qin YW and Jing Q: Circulating microRNA: A novel potential biomarker for early diagnosis of acute myocardial infarction in humans. Eur Heart J 31: 659-666, 2010.
48. Zhang L, Chen X, Su T, Li H, Huang Q, Wu D, Yang C and Han Z: Circulating miR-499 are novel and sensitive biomarker of acute myocardial infarction. J Thorac Dis 7: 303-308, 2015.

49. Hosoda T, Zheng H, Cabral-da-Silva M, Sanada F, Ide-Iwata N Ogórek B, Ferreira-Martins J, Arranto C, D'Amario D, del Monte F, et al: Human cardiac stem cell differentiation is regulated by a mircrine mechanism. Circulation 123: 1287-1296, 2011. 